\title{
Improving the management of T1 bladder cancer
}

The search continues for an accurate method of identifying patients with non-muscle-invasive bladder cancer who are most likely to require radical cystectomy. The best factors on which to base prognosis are unclear-size, tumor multiplicity, carcinoma in situ (CIS), and substage have all been suggested to predict disease progression. Data from two recently published studies shed some light on the matter.

Bas van Rhijn and colleagues propose a new substaging system for T1 tumors, which performs better than the frequently used T1a-c classification. The latter is based on the extent of disease invasion into the muscularis mucosae-vascular plexus (MM-VP), which can be difficult to identify. Instead, van Rhijn et al. suggest defining T1 tumors as microinvasive (T1m; single focus of lamina propria invasion with diameter $\leq 0.5 \mathrm{~mm}$ ) or extensive-invasive (T1e; single focus of lamina propria invasion with diameter $>0.5 \mathrm{~mm}$ or multiple microinvasive areas).
Investigators at centers in The Netherlands and Canada compared the two substaging systems retrospectively in 134 patients with $\mathrm{T} 1$ bladder cancer, all of whom were initially managed conservatively with BCG therapy and followed up for a median of 6.4 years. Primary transurethral resection specimens were staged by a single pathologist using both methods.

The new system could predict clinical outcome better than the T1a-c system. On multivariate analysis, $\mathrm{T} 1 \mathrm{~m} / \mathrm{T} 1 \mathrm{e}$ stage was a significant predictor of both progression $(P=0.001)$ and disease-specific survival $(P=0.032)$, whereas staging according to T1a-c was not. Female gender $(P=0.006)$ and CIS $(P=0.034)$ were also significantly associated with progression.

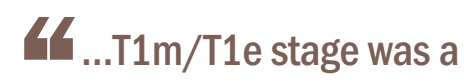
significant predictor of both progression ... and diseasespecific survival... 77
The authors hope that validation of their system in other cohorts and by other pathologists will follow, and that substage according to $\mathrm{T} 1 \mathrm{~m} / \mathrm{T} 1 \mathrm{e}$ will ultimately be incorporated into the TNM classification system.

The second study, from a single center in Spain, of 146 patients with T1G3 bladder cancer treated with BCG, revealed that CIS in the prostatic urethra was a prognostic factor for progression $(P=0.001)$. The authors emphasize the importance of performing a biopsy of the prostatic urethra in patients with high-grade disease for prognostic purposes.

\section{Sarah Payton}

Original articles van Rhijn, B. W. G. et al. A new and highly prognostic system to discern T1 bladder cancer substage. Eur. Urol. doi.10.1016/j.eururo.2011.10.026 | Palou, J. et al. Female gender and carcinoma in situe in the prostatic urethra are prognostic factors for recurrence, progression, and disease-specific mortality in T1G3 bladder cancer patients treated with bacillus calmette-guérin. Eur. Urol. doi:10.1016/j.eururo.2011.10.029 